\title{
In-line Critical Dimension Measurement System Development of LCD Pattem Proposed by Newly Developed Edge Detection Algorithm
}

\author{
Sung-Hoon Park ${ }^{1}$, Jeong-Ho Lee ${ }^{2 *}$, and Heui-Jae Pahk ${ }^{1}$ \\ ${ }^{I}$ School of Mechanical and Aerospace Engineering, Seoul National University, Seoul 151-744, Korea \\ ${ }^{2}$ Optical Equipment Group, Samsung Electro-Mechanics, 314, Maetan 3-dong, Yeongtong-gu, Suwon \\ 443-743, Korea
}

(Received June 20, 2013 : revised August 1, 2013 : accepted August 27, 2013)

\begin{abstract}
As the essential techniques for the CD (Critical Dimension) measurement of the LCD pattern, there are various modules such as an optics design, auto-focus [1-4], and precise edge detection. Since the operation of image enhancement to improve the $\mathrm{CD}$ measurement repeatability, a ring type of the reflected lighting optics is devised. It has a simpler structure than the transmission light optics, but it delivers the same output. The edge detection is the most essential function of the $\mathrm{CD}$ measurements. The $\mathrm{CD}$ measurement is a vital inspection for LCDs [5-6] and semiconductors [7-8] to improve the production yield rate, there are numbers of techniques to measure the CD. So in this study, a new subpixel algorithm is developed through facet modeling, which complements the previous sub-pixel edge detection algorithm. Currently this CD measurement system is being used in LCD manufacturing systems for repeatability of less than $30 \mathrm{~nm}$.
\end{abstract}

Keywords: CD (Critical dimension), Facet modeling, Subpixel edge detection

OCIS codes : (100.0100) Image processing; (110.2960) Image analysis; (120.3940) Metrology; (150.3045) Industrial optical metrology

\section{INTRODUCTION}

In accordance with a trend of TFT-LCD panel's enlargement, the requirement of $\mathrm{CD}$ measurement accuracy for each process of TFT-LCD manufacture is getting stricter. In order to improve the contrast of edges for the CD measurement, reflected light type optics is devised and applied, that has the same effect as with transmission light. Finally, a new sub-pixel algorithm is developed which complements the previously proposed sub-pixel algorithm for edge detection. We applied this system to the $\mathrm{CD}$ measurement process, and analyzed the results.

\section{DESIGNED OPTICAL SYSTEM}

Figure 1 shows the devised system. This system consists of the ring type of reflected light which has the same effect as with the transmission light, lens, glass surface plate, aluminum surface plate, reflected sheet between glass and aluminum surface plates and a camera as an imager.

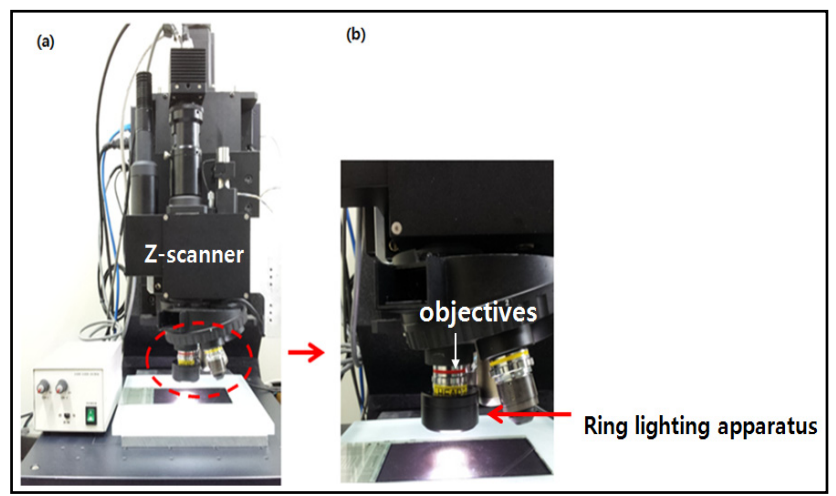

FIG. 1. CD measurement System; (a) CD measurement optics, (b) The lens with attached ring lighting apparatus.

\footnotetext{
*Corresponding author: author: ejsilk01@gmail.com

Color versions of one or more of the figures in this paper are available online.
} 


\section{RING TYPE OF THE REFLECTED LIGHT OPTICS}

The difference of intensity value around the edge between the object and the background is a very important factor in the $\mathrm{CD}$ measurement. It affects the $\mathrm{CD}$ measurement as well as the calculation of the focus position. In the $\mathrm{CD}$ measurements system, the light apparatus is divided into

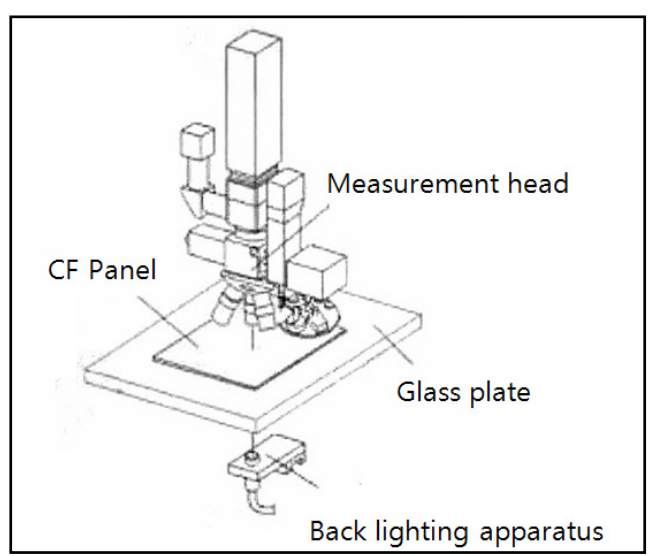

FIG. 2. Measurement system of transmission lighting type. two types. The first is reflected light apparatus and the other is transmission light apparatus. Usually reflected light optics are useful to inspect the overall appearance of the inside of objects, but transmission light is more useful to improve the contrast of edge for CD measurement. However there are disadvantages of applying the in-line $\mathrm{CD}$ measurement system using transmission light. For example, the transmission light apparatus and optics must be in the opposite direction based on the glass plate. Also the system using transmission light must use fragile materials such as glass for the sample support.

In addition, the transmission light system has more complicated structure compared to the reflected light system, as the optics and transmission light under the glass plate must be moved in synchronization. So in this study, we devised a system which is a reflected light type, but has the same effect as with the transmission light. The designed optical system is shown in Fig. 3.

Figures 4(a) and 4(b) show the captured overlay pattern images by transmission light optics and the designed ring light optics respectively. No significant difference was found.

In addition, the unnecessary marks that can potentially cause errors in $\mathrm{CD}$ measurement of normal coaxial reflected light optics were remarkably reduced in the devised ring light type optics. (Fig. 5)

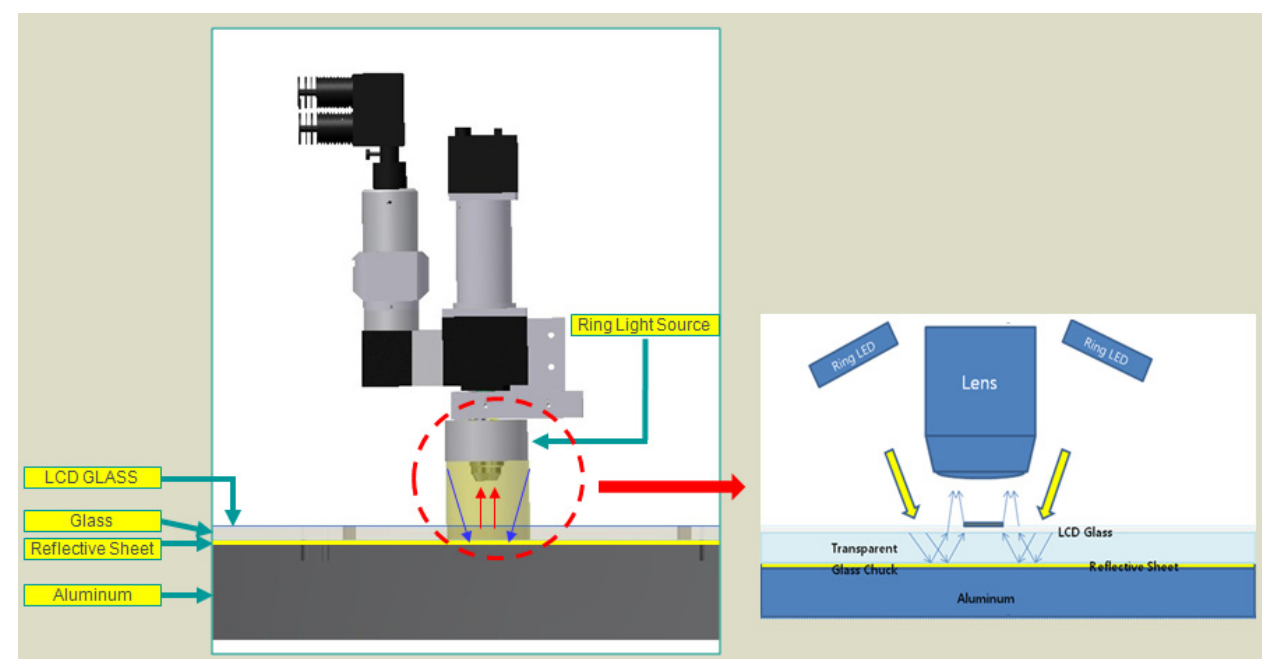

FIG. 3. Measurement head of ring (reflection) light type.
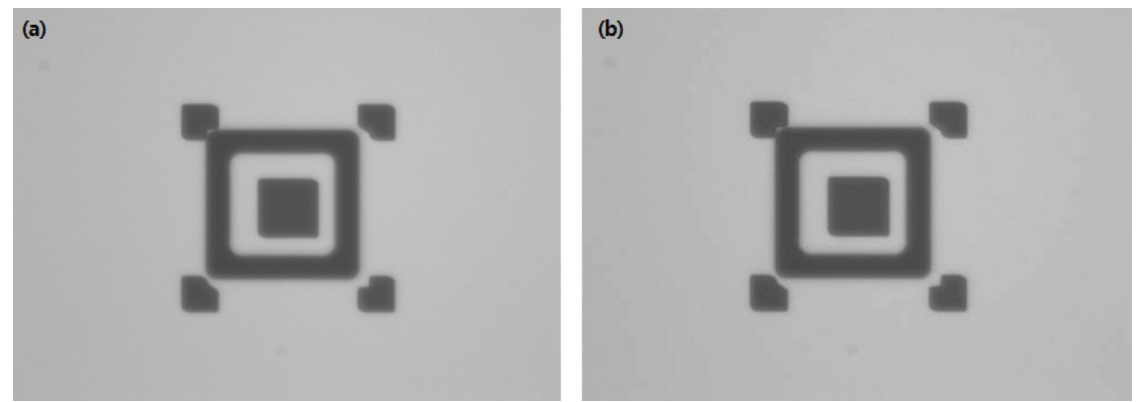

FIG. 4. Overlay image by transmission and ring (reflection) light; (a) Back lighting (transmission type), (b) Ring lighting type (reflection type). 


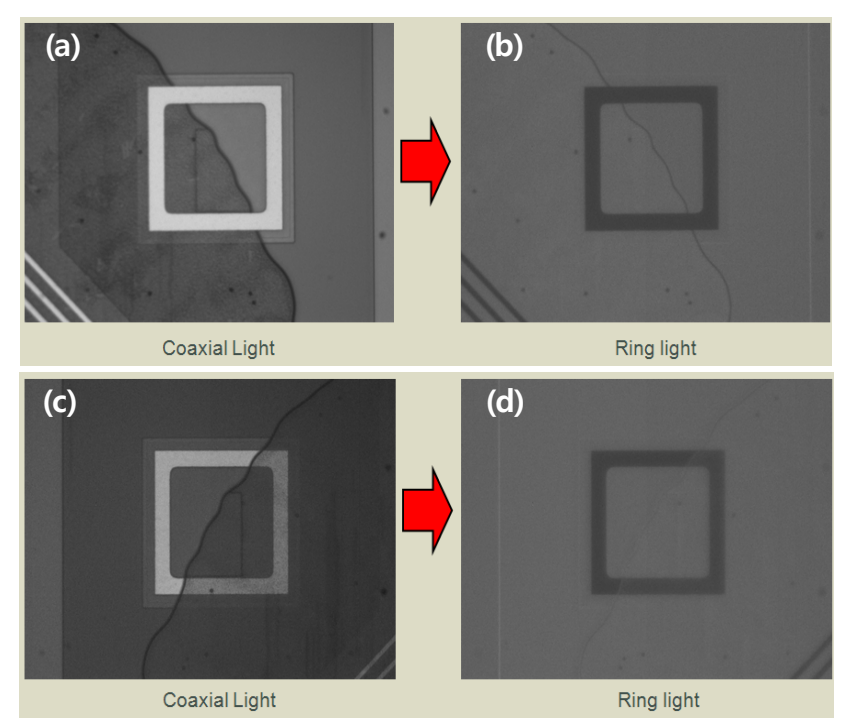

FIG. 5. TFT- LCD patterns; (a) TFT upper side coaxial light,

(b) TFT upper side ring light, (c) CF upper side coaxial light,

(d) CF upper side ring light.

\section{EDGE DETECTION ALGORITHM}

Edge detection is the preprocessing level of images for $\mathrm{CD}$ measurement. It senses the change of light and shade, then distinguishes the object from the background. There are Sobel, Laplacian, LoG(Laplacian of Gaussian) and Canny edge detectors that have the accuracy of the pixel level. Based on the results extracted by edge detection of pixel level, it is possible to detect the edge at the subpixel level, such as by interpolation and by the method that models the intensity of images by a parametric model[9-10]. In this study, we first detected the edge by the LoG operator of the pixel level and then conducted the subpixel level detection using facet modeling.

\subsection{LoG Operator}

The edge of an object is a very important clue as it can connect an image with its interpretation. Edge detection is conducted usually by convolution that uses the surrounding pixels of a narrow area, as in work by Kirsch and Sobel. These operators are easy to use and calculate quickly, but are very sensitive to noise and highly dependent on the magnitude of objects. To compensate for these disadvantages, the operator of edge detection by zero-crossing of second order derivative was applied. This is based on the fact that the result of the first order derivative has the maximum value in the edge of the image, and the result of the second order derivative is zero on the same spot. To get the second order derivative figure of the image, we employed a smoothing filtering. This process reduces noise. The smoothing filter here that we used is $2 \mathrm{D}$ Gaussian $\mathrm{G}(\mathrm{x}, \mathrm{y})$.

$$
G(x, y)=e^{-\frac{x^{2}+y^{2}}{2 \sigma^{2}}}
$$

$x, y$ are the image coordinates, $\sigma$ is the standard deviation of the probability distribution. Gaussian function is often used as normalized form. The normalized Gaussian function is as follow [11].

$$
G(x, y)=\frac{1}{2 \pi \sigma^{2}} e^{-\frac{x^{2}+y^{2}}{2 \sigma^{2}}} \text { or } G(x, y)=\frac{1}{\sqrt{2 \pi} \sigma} e^{-\frac{x^{2}+y^{2}}{2 \sigma^{2}}}
$$

In the above equation, $\sigma$ is the standard deviation of the probability distribution. The standard deviation $\sigma$ is proportional to the size of the Gaussian operator, and as the pixels get far away from the center of the Gaussian standard deviation, they have less influence. And the pixel farther from $3 \sigma$ of the Gaussian center has almost no influence. The second derivative image reduced by the Gaussian operator can be obtained through the Laplacian operator. The form of a Laplacian of Gaussian (LoG) is as follows.

$$
\nabla^{2}[G(x, y, \sigma) * f(x, y)]
$$

Due to the linearity of the LoG, the convolution and differential operation can be interchangeable. The form is as follows.

$$
\left[\nabla^{2} G(x, y, \sigma)\right] * f(x, y)
$$

The above equation $\nabla^{2} G(x, y)$ can be calculated in advance, because it is independent of the image. The form of $\nabla^{2} G(x, y)$ is as follows [12]:

$$
\nabla^{2} G(x, y)=K\left(2-\left(\frac{\left.x^{2}+y^{2}\right)}{\sigma^{2}}\right)\right) \bullet \exp \left[-\frac{\left(x^{2}+y^{2}\right)}{2 \sigma^{2}}\right]
$$

$\mathrm{K}$ is a scale factor and makes the sum of all LoG components zero. When $\sigma=\sqrt{2}, K=4232$, the $15 \times 15 \mathrm{LoG}$ is as follows:

$$
\left[\begin{array}{rrrrrrrr}
0 & 0 & 0 & 0 & 0 & 0 & 0 & 0 \\
0 & 0 & 0 & 0 & -1 & -2 & -3 & -4 \\
0 & 0 & 0 & -1 & -6 & -19 & -35 & -43 \\
0 & 0 & -1 & -10 & -43 & -114 & -196 & -233 \\
0 & -1 & -6 & -43 & -165 & -369 & -521 & -558 \\
0 & -2 & -19 & -114 & -369 & -573 & -303 & 0 \\
0 & -3 & -35 & -196 & -521 & -303 & 1282 & 2472 \\
0 & -4 & -43 & -233 & -558 & 0 & 2472 & 4232
\end{array}\right]
$$

FIG. 6. Laplacian Gaussian filter (upper left quadrant). 


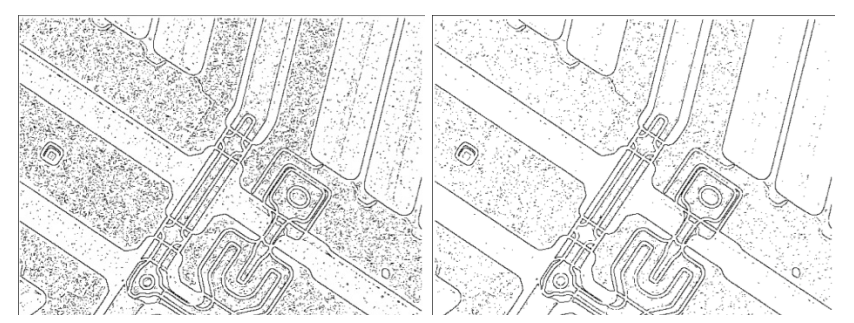

(a)

(b)

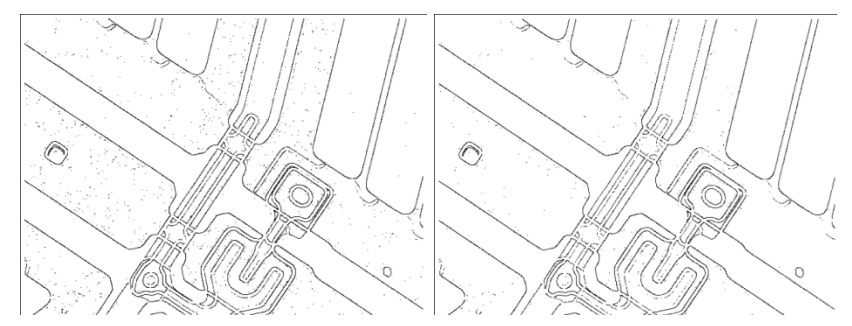

(c)

(d)

FIG. 7. Edge image of TFT sample using original LoG filter; (a) Threshold is $20 \%$ of Max. edge magnitude, (b) Threshold is $50 \%$ of Max. edge magnitude, (c) Threshold is $70 \%$ of Max. edge magnitude, (d) Threshold is $90 \%$ of Max. edge magnitude.

This is the upper left part of the matrix because it has a symmetry structure up and down and right and left. We conducted the edge detection for the sample image of TFT-LCD, using the LoG from the above. While we detected the edge, we changed the threshold by $90 \%, 70 \%$, $50 \%$ and $20 \%$ of the edge's maximum size. The result shows that the higher we make the threshold, the smaller noise edges appear.

\subsection{Subpixel Edge Detection}

In general, there are two types of subpixel edge detection methods, the parametric model method [10] and interpolation by interpolating the intensity of the edge. In the parametric model, there is one method of edge detection with subpixel precision after modeling under the assumption that the change of image intensity is a continuous function. Another method is to get a boundary of zero-crossing with subpixel precision. This process is followed by getting a boundary of zerocrossing with pixel precision by LoG and then gets a continuous function with the assumption that the surrounding data of zero-crossing are fitted as a facet model.

\subsubsection{Interpolation}

The sub-pixel edge detection using the interpolation method is as follows. First it extracts the edge at pixel level, and calculates the strength of the edge. Then, it extracts the edge at subpixel level using the parabolic or Gaussian modeling. This method has a precondition that the strength of the edge should be changed continuously and smoothly, also the surrounding area of maximum value of the edge can be modeled as parabolic or Gaussian modeling [14].

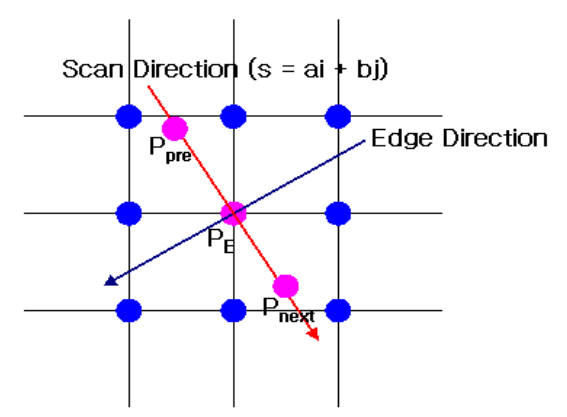

FIG. 8. Interpolation for subpixel edge detection.

If the pixel level edge $P_{E}$ has the edge strength $C_{E}$, from $\left(-1, C_{P R E}\right),\left(0, C_{E}\right),\left(1, C_{N E X T}\right)$, the position of the maximum value can be calculated by parabolic or Gaussian modeling. The position calculated by the parabolic modeling is as follows:

$$
P_{s u b E}=P_{E}+\frac{C_{p r e}-C_{n e x t}}{2\left(C_{p r e}+C_{n e x t}-2 C_{E}\right)} \cdot(a i+b j)
$$

As for the Gaussian modeling, we assume its function as follows:

$$
G(x)=C \exp \left[-\left(x_{0}-x\right)^{2} / k\right]
$$

When we substituted $\left(-1, C_{P R E}\right),\left(0, C_{E}\right),\left(1, C_{N E X T}\right)$ into function (7) and took the natural logarithm, the result was as follows:

$$
\begin{aligned}
& \ln G(-1)=\ln C_{\text {prev }}=\ln C+\frac{\left(-x_{0}-(-1)\right)^{2}}{k}=\ln C+\frac{\left(x_{0}{ }^{2}+2 x_{0}+1\right)}{k} \\
& \ln G(0)=\ln C_{E}=\ln C+\frac{\left(x_{0}\right)^{2}}{k} \\
& \ln G(-1)=\ln C_{\text {next }}=\ln C+\frac{\left(x_{0}-1\right)^{2}}{k}=\ln C+\frac{\left(x_{0}{ }^{2}-2 x_{0}+1\right)}{k}
\end{aligned}
$$

Using (8), (9) and (10), equation (11) is obtained.

$$
\ln G(-1)+\ln G(1)-2 \ln G(0)=\ln C_{\text {prev }}+\ln C_{E}+\ln C_{\text {next }}=\frac{2}{k}
$$

$x_{0}=\frac{\ln C_{\text {pre }}-\ln C_{\text {next }}}{2 \ln C_{\text {pre }}-4 \ln C_{E}+2 \ln C_{\text {next }}}$ is the result of equation (8) subtracting (9) and dividing by (11). Here $x_{0}$ means the difference between $P_{E}$ and $P_{\text {subE }}$ (since the intensity of pixel at $\mathrm{x}=0$ is $P_{E}$ ). So we can describe the equation (12), $P_{s u b}$. 


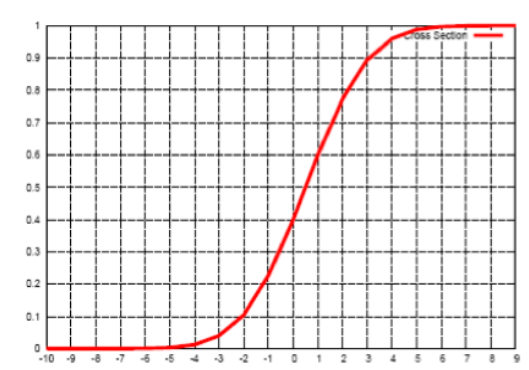

(a)

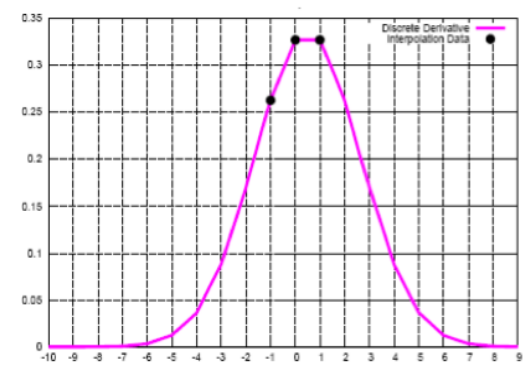

(b)

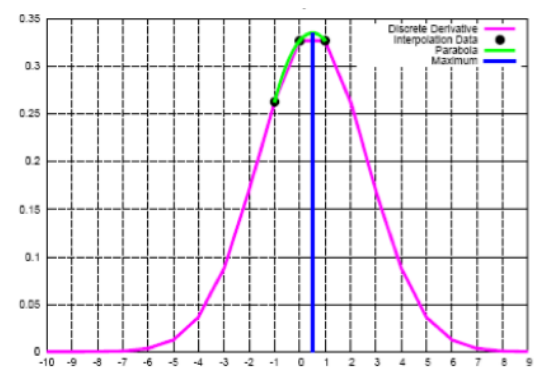

(c)

FIG. 9. Parabolic interpolation for subpixel edge detection; (a) $f(x)=\operatorname{erf}(x-0.5)$, (b) Discrete derivative, (c) Parabolic interpolation.

$$
P_{\text {subE }}=P_{E}+\frac{\ln C_{p r e}-\ln C_{n e x t}}{2 \ln C_{p r e}-4 \ln C_{E}+2 \ln C_{n e x t}} \cdot(a i+b j)
$$

Edge detection by sub-pixel interpolation takes less time because its calculation is relatively simple. But the strength of the edge does not change continuously and smoothly all the time, it may be changed rapidly. So it may be inaccurate to apply a parabolic or Gaussian modeling.

\subsubsection{Parametric Model}

The parametric modeling begins with the idea that in the local area of images, the intensity value can be assumed as a continuous function [15]. It is impossible to express the changes of images' intensity as a single continuous function, so we obtain a local continuous function called the facet model, with the surrounding pixels in a certain area. Using this continuous function, the intensity value of images at subpixel level can be obtained. The algorithm by the facet model is as follows.

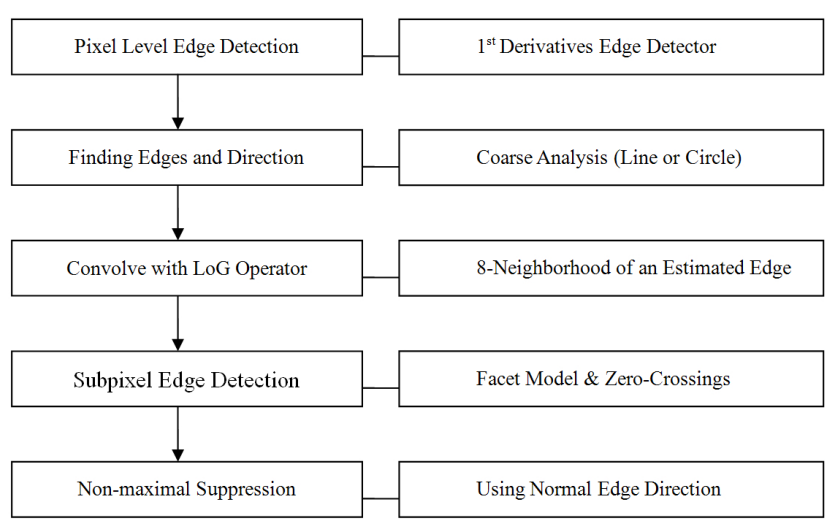

FIG. 10. Procedure of subpixel edge detection using facet model.

As shown in the above algorithm, first we calculate the convolution $I_{L o G}$ of Image I by LoG operator. And then, search zero-crossings point within the $I_{L o G}$. Around each of the zero-crossings, a continuous function can be modeled by the facet model. In this study, the facet model is as follows:

$$
\begin{aligned}
I_{L o G}(r, c) & =k_{0}+k_{1} r+k_{2} c+k_{3} r^{2} \\
& +k_{4} r c+k_{5} c^{2}+k_{6} r^{2} c+k_{7} c^{2} r+k_{8} r^{2} c^{2}
\end{aligned}
$$

where $-1 \leq r, c \leq 1$.

When the facet model can be defined as above, the direction of the edge can be obtained as follows.

$$
\theta=\tan ^{-1}\left[\frac{k_{2}}{k_{3}}\right]
$$

Since $I_{L o G}(r, c)$ is a continuous function, which can be expressed as the sum of the orthogonal polynomials, the equation is as follows:

$$
I_{L o G}(r, c)=\sum_{n=1}^{N} a_{n} P_{n}(r, c)
$$

$P_{n}(r, c)$ means the set of Chebyshev discrete orthogonal polynomials. $a_{n}$ is the weighting coefficients. $a_{n}$ can be calculated as follows.

$$
\begin{aligned}
& a_{n}=\frac{\sum_{r} \sum_{c} P_{n}(r, c) I_{L o G}(r, c)}{\sum_{i} \sum_{j} P_{n}^{2}(i, j)} \\
& p_{0}(r, c)=1 \\
& p_{1}(r, c)=2 \\
& p_{2}(r, c)=c \\
& p_{3}(r, c)=r^{2}-2 / 3
\end{aligned}
$$




$$
\begin{aligned}
& p_{6}(r, c)=c\left(r^{2}-2 / 3\right) \\
& p_{7}(r, c)=r\left(c^{2}-2 / 3\right) \\
& p_{8}(r, c)=\left(r^{2}-2 / 3\right)\left(c^{2}-2 / 3\right)
\end{aligned}
$$

The weighting coefficients $a_{n}$ can be obtained from the above equation. Between polynomial coefficient $k_{n}$ and weighting coefficients $a_{n}$ that are previously used in the facet model, the following relation equation has been established.

$$
\begin{aligned}
& k_{0}=a_{0}-(2 / 3) a_{3}-(2 / 3) a_{5}+(4 / 9) a_{8} \\
& k_{1}=a_{1}-(2 / 3) a_{6} \\
& k_{2}=a_{2}-(2 / 3) a_{7} \\
& k_{3}=a_{3}-(2 / 3) a_{8} \\
& k_{4}=a_{4} \\
& k_{5}=a_{5}-(2 / 3) a_{8} \\
& k_{6}=a_{6} \\
& k_{7}=a_{7} \\
& k_{8}=a_{8}
\end{aligned}
$$

Therefore, in order to obtain the facet model, we suggest not to obtain a polynomial coefficient directly, but to obtain the weighting coefficients $a_{n}$ first, and then get the $k_{n}$ by using the above equation. And after we get the facet model, $I_{L o G}(r, c)$ can be calculated with the $n$ level of subpixel precision.

$$
\{r, c\}=\left\{-1,-\frac{n / 2-1}{n / 2},-\frac{n / 2-2}{n / 2}, \ldots,-\frac{1}{n / 2}, 0, \frac{1}{n / 2}, \ldots, \frac{n / 2-2}{n / 2}, \frac{n / 2-1}{n / 2}, 1\right\}
$$

\section{ANALYSIS}

In this study, a sample pattern was produced in the process of TFT-LCD. The distance between the patterns was measured 20 times.
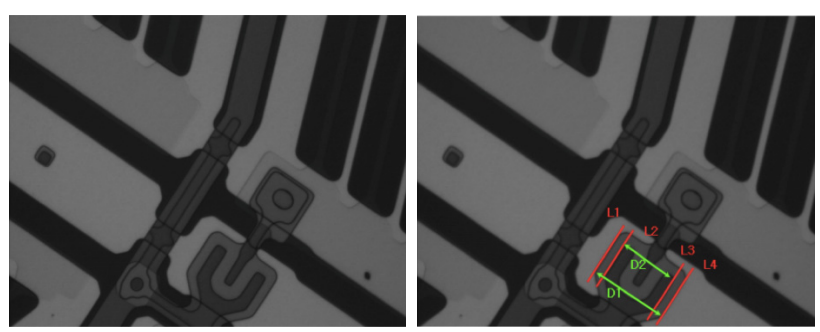

FIG. 11. TFT-LCD sample image and CD.

D1 : The distance between the L1 and L4

D2 : The distance between the L2 and L3
- Pixel level edge detection

TABLE 1. TFT CD measurement result using pixel level edge detection (unit: um)

\begin{tabular}{c|c|c||c|c|c}
\hline \hline No. & Distance1 & Distance2 & No. & Distance1 & Disance2 \\
\hline 1 & 36.6170 & 27.5780 & 11 & 36.6179 & 27.5772 \\
\hline 2 & 36.5856 & 27.5711 & 12 & 36.6256 & 27.5777 \\
\hline 3 & 36.6238 & 27.5995 & 13 & 36.5952 & 27.5774 \\
\hline 4 & 36.6363 & 27.5634 & 14 & 36.5857 & 27.5134 \\
\hline 5 & 36.6579 & 27.4901 & 15 & 36.6198 & 27.5780 \\
\hline 6 & 36.6542 & 27.5517 & 16 & 36.6230 & 27.4848 \\
\hline 7 & 36.6294 & 27.6475 & 17 & 36.6115 & 27.5634 \\
\hline 8 & 36.6368 & 27.5708 & 18 & 36.6149 & 27.5918 \\
\hline 9 & 36.6562 & 27.5674 & 19 & 36.6137 & 27.5932 \\
\hline 10 & 36.6115 & 27.5773 & 20 & 36.6176 & 27.4590 \\
\hline Average & 36.6217 & 27.5616 & & \multicolumn{3}{l}{} \\
\hline 3Sigma & 0.0606 & 0.1314 & & &
\end{tabular}

- Subpixel level edge detection using parabolic interpolation

TABLE 2. TFT CD measurement result using parabolic interpolation (unit: um)

\begin{tabular}{c|c|c||c|c|c}
\hline \hline No. & Distance1 & Distance2 & No. & Distance1 & Disance2 \\
\hline 1 & 36.5386 & 27.4724 & 11 & 36.5198 & 27.4878 \\
\hline 2 & 36.5176 & 27.4915 & 12 & 36.5288 & 27.4724 \\
\hline 3 & 36.5369 & 27.4805 & 13 & 36.5396 & 27.4938 \\
\hline 4 & 36.5318 & 27.4690 & 14 & 36.5659 & 27.4538 \\
\hline 5 & 36.5549 & 27.4745 & 15 & 36.5412 & 27.4806 \\
\hline 6 & 36.5299 & 27.4518 & 16 & 36.5566 & 27.4864 \\
\hline 7 & 36.5363 & 27.4682 & 17 & 36.5384 & 27.4837 \\
\hline 8 & 36.5309 & 27.4671 & 18 & 36.5897 & 27.5087 \\
\hline 9 & 36.5286 & 27.4624 & 19 & 36.5513 & 27.4806 \\
\hline 10 & 36.5779 & 27.4402 & 20 & 36.5406 & 27.4742 \\
\hline Average & 36.5428 & 27.4750 & \multicolumn{4}{|c}{} \\
\hline 3Sigma & 0.0555 & 0.0474 & \multicolumn{4}{|c}{} \\
\hline
\end{tabular}

- Subpixel level edge detection using facet model

TABLE 3. TFT CD measurement result using facet model (unit: um)

\begin{tabular}{c|c|c||c|c|c}
\hline \hline No. & Distance1 & Distance2 & No. & Distance1 & Disance2 \\
\hline 1 & 36.5841 & 27.5229 & 11 & 36.5885 & 27.5305 \\
\hline 2 & 36.5863 & 27.5249 & 12 & 36.5943 & 27.5289 \\
\hline 3 & 36.5858 & 27.5190 & 13 & 36.5771 & 27.5158 \\
\hline 4 & 36.5877 & 27.5336 & 14 & 36.5829 & 27.5176 \\
\hline 5 & 36.5714 & 27.5260 & 15 & 36.5937 & 27.5412 \\
\hline 6 & 36.5924 & 27.5416 & 16 & 36.5954 & 27.5367 \\
\hline 7 & 36.5841 & 27.5267 & 17 & 36.5889 & 27.5335 \\
\hline 8 & 36.5855 & 27.5247 & 18 & 36.5903 & 27.5334 \\
\hline 9 & 36.5835 & 27.5208 & 19 & 36.5806 & 27.5224 \\
\hline 10 & 36.5932 & 27.5246 & 20 & 36.5840 & 27.5257 \\
\hline Average & 36.5865 & 27.5275 & & & \\
\hline 3Sigma & 0.0181 & 0.0220 & & & \\
\hline
\end{tabular}




\section{CONCLUSION}

In this study, we conducted research to develop a $\mathrm{CD}$ measurement algorithm of more stable and accurate TFTLCD patterns, and the conclusion is as below.

1. We built a ring type of the reflected light optics for $\mathrm{CD}$ measurement and installed it in the stage.

2. For more accurate and high-speed measurement, we detected the edge's accuracy of pixel level first, and detected the edge around the detected ones again of subpixel level.

3. We used the first differential operator for the edge detection of pixel level. For the edge detection of subpixel accuracy, we modeled the detected edge filtered by LoG with the facet model, and calculated the local intensity continuous function, and obtained the zero-crossing with it.

4. We compared the performances between the proposed subpixel edge detection algorithm and the existing interpolated subpixel edge algorithm, and pixel level edge detection. As a result, it is confirmed that the proposed algorithm can detect the edge most reliably and precisely.

\section{REFERENCES}

1. T. T. E. Yeo, S. H. Ong, Jayasooriah, and R. Sinniah, "Autofocusing for tissue microscopy," Image and Vision Computing 11, 629-639 (1993).

2. D. L. Marks, A. L. Oldenburg, J. J. Reynolds, and S. A. Boppart, "Autofocus algorithm for dispersion correction in optical coherence tomography," Beckman Institute of Advanced Science and Technology 42, 3038-3046 (2003).

3. S. E. Kornegay and M. Dagenais, "Focus and edge detection algorithms and their relevance to the development of an optical overlay calibration standard," Proc. SPIE 3677, 95-106 (1999).

4. J.-H. Ahn, J. Ko, I.-Y. Lee, and S.-H. Kim, "A fast continuous auto focus algorithm using the state transition model," Pacific Science Review 13, 125-130 (2011).

5. N.-T. Doan, J. H. Moon, T.-W. Kim, and H.-J. Pahk, "A fast image enhancement technique using a new scanning path for critical dimension measurement of glass panels," International Journal of Precision Engineering and Manufacturing 13, 2109-2114 (2012).

6. W. Lee, S. H. Han, and H. Jeong, "Measurement of critical dimension in scanning electron microscope mask images," J. Micro/Nanolith, MEMS and MOEMS 10, 023003 (2011).

7. J.-Y Hur and M.-S. Seo, "Optical proximity corrections for digital micro mirror device-based maskless lithography," J. Opt. Soc. Korea 16, 221-227 (2012).

8. B. H. Nam and H. J. Lee, "Gate CD control for memory chip using total process proximity based correction method," J. Opt. Soc. Korea 6, 180-184 (2002).

9. A. Huertas and G. Medioni, "Detection of intensity changes with subpixel accuracy using laplacian-gaussian masks," IEEE Trans. Pattern Analysis and Machine Intelligence 8, 651-664 (1986).

10. R. M. Haralick and L. G. Shapiro, Computer and Robot Vision, Vol. I (Addison-Wesley, Reading, MA, USA, 1992).

11. M. Sonka, V. Hlavac, and R. Boyle, Image Processsing, Analysis, and Machine Vision Sencond Edition (PWS Publishing, 1999), p. 84.

12. D. Marr and E. Hildreth, "Theory of edge detection," Proc. Roy. Soc. London B207, 187-217 (1980).

13. R. M. Haralick, "Digital step edge from zero crossing of second directional derivatives," IEEE Computer Society 6, 58-68 (1984).

14. H. Nobach, N. Damaschke, and C. Tropea, "High-precision sub-pixel interpolation in particle image velocimetry image processing," Experiments in Fluids 39, 299-304 (2005).

15. R. Nevatial, "Evaluation of a simplified Hueckel edge-line detector," Computer Graphics and Image Processing 6, 582-588 (1977). 